Sebastian Schädel*, Anita Przyklenk, Martin Stein, Karin Kniel und Eberhard Manske

\title{
Ganzheitliche Kalibrierung von Gewinden auf Basis eines dreidimensionalen Ansatzes
}

\author{
Holistic calibration of screw threads based on a three-dimensional approach
}

\section{DOI 10.1515/teme-2020-0035}

Zusammenfassung: Gewinde sind Maschinenelemente mit vielseitiger Funktionalität. Sie werden zum Befestigen, Verbinden, Abdichten oder Zentrieren verwendet und in großen Stückzahlen hergestellt. Dabei steigen die Anforderungen an die Fertigung und Qualitätssicherung stetig an. Nach aktuellen Normen und Richtlinien erfolgt die Prüfung der Bestimmungsgrößen an Gewinden stichprobenartig in wenigen festgelegten Axialschnitten und Prüfebenen. Die komplex geformten und stark gekrümmten Werkstückoberflächen von Gewindeflanken werden deshalb hinsichtlich ihrer Funktionalität unzureichend bewertet. Das neuartige Messverfahren basiert auf einem dreidimensionalen mathematischen Modell zur Beschreibung der Gewindegeometrie. Die Aufnahme der Messpunkte als IstPunktewolke erfolgt durch eine flächenhafte Messstrategie auf konventionellen Koordinatenmessgeräten. Der anschließende ganzheitliche Auswertealgorithmus ermöglicht erstmals die Bestimmung aller relevanten Bestimmungsgrößen am Gewinde auf Basis eines einzigen Datensatzes. Des Weiteren liefert der neue Auswertealgorithmus erstmals ein Ergebnis für die lokalen Bestimmungsgrößen, welche zur Berechnung des Paarungsflankendurchmessers benötigt werden.

Schlüsselwörter: Gewindemetrologie, 3D-Koordinatenmesstechnik, flächenhafte Messstrategie, ganzheitlicher Auswertealgorithmus, lokale Bestimmungsgrößen.

Abstract: Screw threads are important machine elements with various functions. They are used for mounting, connecting, sealing or centering of components and are pro-

*Korrespondenzautor: Sebastian Schädel, ZEISS Industrial Quality Solutions, Carl Zeiss Industrielle Messtechnik GmbH, CarlZeiss-Straße 22, 73446 Oberkochen, E-Mail:

sebastian.schaedel@zeiss.com

Anita Przyklenk, Martin Stein, Karin Kniel, Fachbereich Koordinatenmesstechnik, Physikalisch-Technische Bundesanstalt, Bundesallee 100, 38116 Braunschweig, E-Mail:

anita.przyklenk@ptb.de, martin.stein@ptb.de, karin.kniel@ptb.de Eberhard Manske, Institut für Prozessmess- und Sensortechnik, Technische Universität IImenau, Gustav-Kirchhoff-Straße 1, 98693 Ilmenau, E-Mail: eberhard.manske@tu-ilmenau.de duced in large quantities. At the same time the requirements for manufacturing processes and quality assurance are increasing constantly.

In accordance with current standards the inspection of screw thread determinants is performed in single axial sections. Therefore, the complex geometry of the flanks is not assessed sufficiently with respect to the functionality of the flanks. The novel measurement procedure is based on a three-dimensional mathematical model which describes the screw thread geometry. The actual point cloud of the rough shape is gathered by an areal-oriented measuring strategy using a conventional coordinate measuring machine. The following holistic evaluation algorithm ensures the calculation of all relevant screw thread determinants based on a single data set. Furthermore, the novel measurement procedure provides measurement results of the local screw thread determinants, which are crucial for the calculation of the virtual pitch diameter.

Keywords: Screw thread metrology, 3D-coordinate metrology, areal-oriented measuring strategy, holistic evaluation algorithm, local determinants.

\section{Einleitung}

Gewinde werden gemäß der geltenden Richtlinie [1] bislang nur linienhaft in zwei ausgewählten Axialschnitten gemessen. Auf Grundlage der erfassten Messpunkte werden die Bestimmungsgrößen für das gesamte Gewinde ermittelt. Aus diesem Grund lässt sich mit den momentan verwendeten Messverfahren die Funktion eines Gewindes nur unzureichend bewerten. Des Weiteren fordert der technische Fortschritt im Maschinenbau ein Umdenken in der Gewindemetrologie. Aktuelle Maßtoleranzen im Bereich der Präzisionstechnik lassen sich mit den konventionellen Messverfahren nicht geeignet prüfen. Das Verhältnis von Messunsicherheit zu Maßtoleranz, welches nach der "Goldenen Regel der Fertigungsmesstechnik" zwischen 1/10-1/5 liegen sollte, liegt aktuell bei $1 / 3$, was den Missstand aufzeigt. Eine weitere Reduzierung der Messunsicherheit unter Verwendung des stark limitierten 2D-Messverfahrens 


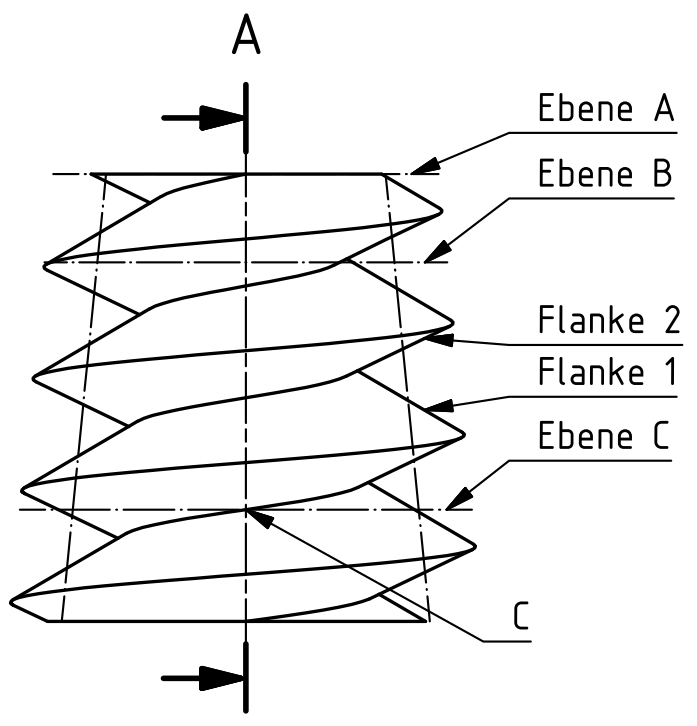

A

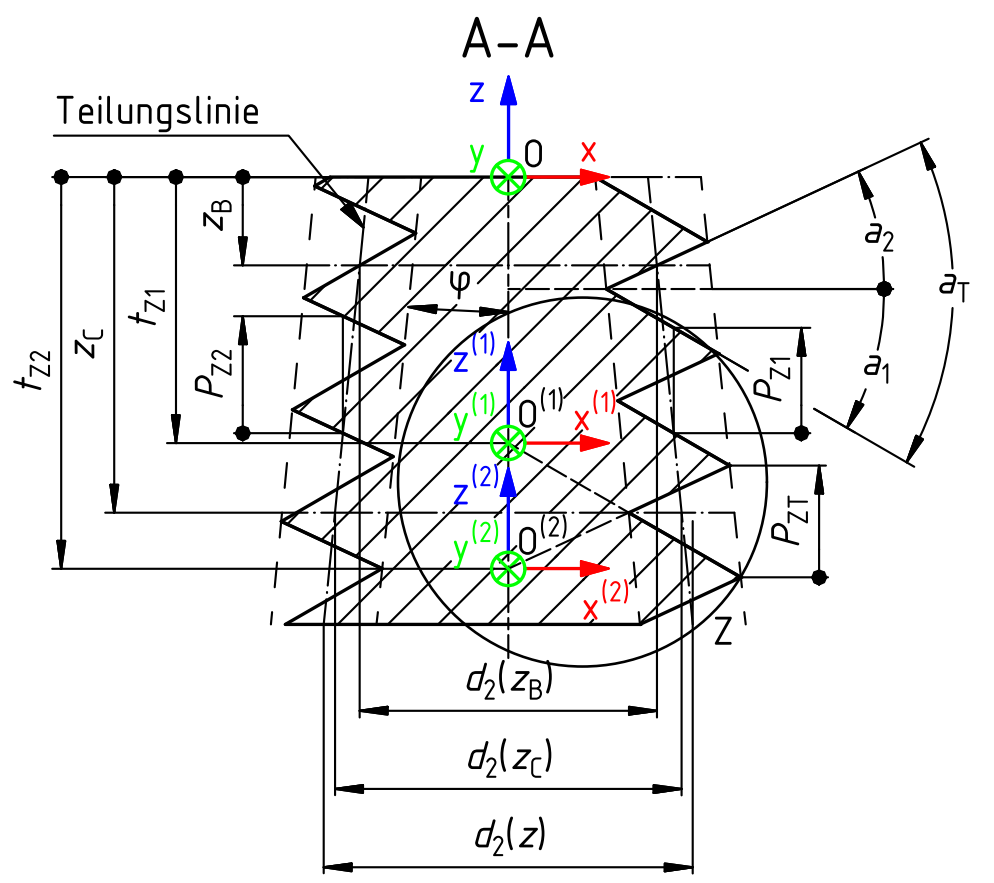

Abb. 1: Geometrisch-ideales Formelement eines Gewindes als Modell der Gewindegeometrie ([8]).

ist nicht möglich. Abhilfe schafft hier eine präzisere und ganzheitliche Betrachtung der Gewindegeometrie unter Verwendung eines 3D-Ansatzes, welcher die Leistungsfähigkeit der modernen 3D-Koordinatenmesstechnik in Gänze ausnutzt.

\section{Modell der Gewindegeometrie}

Das dreidimensionale Modell der Gewindegeometrie in Abb. 1 stellt ein auf Parametern basiertes digitales Abbild der Gewindegeometrie dar und wird in [7] als geometrischideales Formelement bezeichnet. Das geometrisch-ideale Formelement ist so parametrisiert, dass es in die Prozessschritte - Messdatenerfassung in Abschnitt 3 und Messdatenauswertung in Abschnitt 4 - integrierbar ist. Dadurch haben alle notwendigen Prozessschritte zur Ermittlung von Bestimmungsgrößen am Gewinde eine gemeinsame und eindeutige Basis. Ausgehend von der Beschreibung der geometrisch-idealen Ersatzelemente für die Funktionsflächen am Gewinde in Abschnitt 2.1 werden die Eigenschaften des Formelements in Abschnitt 2.2 beschrieben.

\subsection{Regelschraubfläche}

Die Gestalt des Gewindeprofils im Axialschnitt in Abb. 1 entsteht durch das Verschrauben des sogenannten Aus- gangsdreiecks $\triangle \mathrm{CDE}$ in Richtung der z-Achse. Das Ausgangsdreieck $\triangle \mathrm{CDE}$ ist in Detailansicht $\mathrm{Z}$ in Abb. 2 zu sehen. Die Strecken $\overline{\mathrm{CD}}$ und $\overline{\mathrm{CE}}$ des Ausgangsdreiecks $\triangle$ CDE legen als Erzeugende des Gewindeprofils die Form der Funktionsflächen in Richtung der Radialkoordinate $u_{\mathrm{j}}$ fest. Sie sind analytisch durch das Flächenmodell einer Regelschraubfläche exakt beschreibbar. Im geometrisch-idealen Formelement in Abschnitt 2.2 werden die einzelnen Regelschraubflächen in den Hauptachsensystemen $\mathrm{O}^{(\mathrm{j})}$ nach Gl. 1 beschrieben.

$$
S_{\mathrm{j}}\left(u_{\mathrm{j}}, v_{\mathrm{j}}, p_{\mathrm{j} 1}, p_{\mathrm{j} 2}\right)=\left(\begin{array}{c}
x_{\mathrm{j}} \\
y_{\mathrm{j}} \\
z_{\mathrm{j}}
\end{array}\right)=\left(\begin{array}{c}
u_{\mathrm{j}} \cdot \cos \left(v_{\mathrm{j}}\right) \\
u_{\mathrm{j}} \cdot \sin \left(v_{\mathrm{j}}\right) \\
p_{\mathrm{j} 1} \cdot v_{\mathrm{j}}+p_{\mathrm{j} 2} \cdot u_{\mathrm{j}}
\end{array}\right)
$$

Index $\mathrm{j}$ adressiert die voneinander unabhängigen Gewindeflanken. Bei einem eingängigen Gewinde entstehen aus der Verschraubung des Ausgangsdreiecks $\triangle \mathrm{CDE}$ insgesamt zwei Gewindeflanken. Somit wird mit $\mathrm{j}=1$ Flanke 1 und mit $\mathrm{j}=2$ Flanke 2 bezeichnet. Nach [7] ist Flanke 1 diejenige Gewindeflanke am Außengewinde, bei der die z-Komponente des Normalenvektors positiv ist. Flanke 2 beschreibt die Gewindeflanke, bei der die z-Komponente des Normalenvektors negativ ist. Die Parameter $\left(u_{\mathrm{j}}, v_{\mathrm{j}}\right)$ in Gl. 1 werden in der Modellierung allgemein als Flächenparameter und die Parameter $\left(p_{\mathrm{j} 1}, p_{\mathrm{j} 2}\right)$ als Geometrieparameter bezeichnet, wobei $p_{\mathrm{j} 1}$ mit

$$
p_{\mathrm{j} 1}=\frac{P_{\mathrm{Zj}}}{2 \cdot \pi}
$$




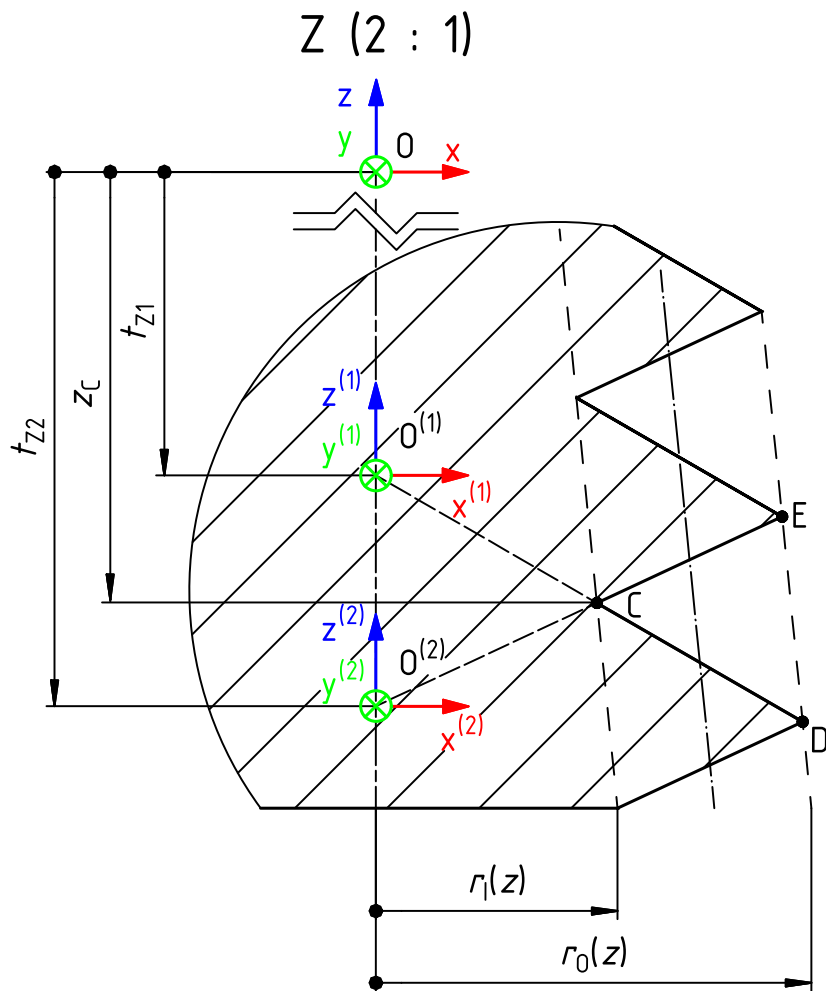

Abb. 2: Detailansicht $Z$ zum Ausgangsdreieck $\triangle \mathrm{CDE}$ und zu den Hauptachsenkoordinatensystemen $\left(\mathrm{O}^{(1)}, \mathrm{O}^{(2)}\right)$ der Regelschraubflächen eines eingängigen Gewindes.

abhängig von der Einzelflankensteigung ist und $p_{\mathrm{j} 2}$ mit

$$
p_{\mathrm{j} 2}=\tan \left(a_{\mathrm{j}}\right)
$$

den Einfluss des Gewindeflankenwinkels $a_{\mathrm{j}}$ (Abb. 1) auf die Gewindegeometrie beschreibt.

\subsection{Geometrisch-ideales Formelement}

Im Umfeld der 2D-Messverfahren haben sich in der Vergangenheit die Ansätze in [2,3] für die Modellierung bzw. die theoretischen Betrachtungen der Gewindegeometrie in der Gewindemetrologie durchgesetzt. Im Vergleich zu [2, 3] stellt Abb. 1 mit der Gesamtheit von Bestimmungsgrößen am Gewinde nach [7] eine deutliche Präzisierung des Modells dar. Am Formelement sind drei Arbeitsebenen zu unterscheiden. Zunächst legt Ebene A als Stirnfläche den Nullpunkt z des Werkstückkoordinatensystems fest. Ebene $\mathrm{B}$ bildet die Prüfebene mit $z_{\mathrm{B}}$ zur Spezifizierung des Flankendurchmessers und Ebene $\mathrm{C}$ ist die Ausgangsebene zur Positionierung des Formelements. Ebene $\mathrm{C}$ enthält den Punkt C, der als Schnittpunkt mit der Koordinate $z_{\mathrm{C}}$ aus den zwei Erzeugenden für die Regelschraubflächen $\overline{\mathrm{CD}}$ und $\overline{\mathrm{CE}}$ entsteht. Mit der Definition der Ausgangsebene $\mathrm{C}$ und dem Schnittpunkt C ist die Position des Ausgangs-

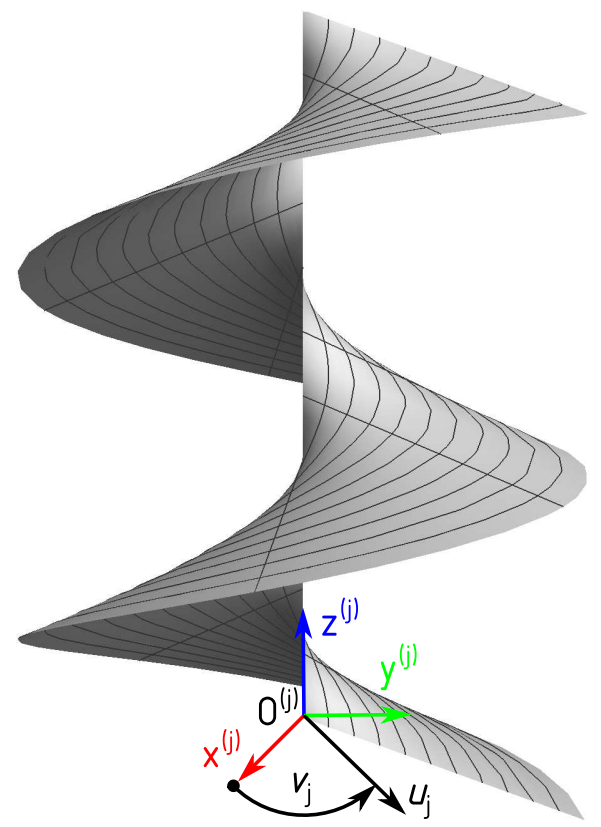

Abb. 3: Regelschraubfläche als geometrisch-ideales Ersatzelement einer Gewindeflanke mit dem kartesischen Hauptachsensystem $\mathrm{O}^{(\mathrm{j})}$ und den Polarkoordinaten $\left(u_{\mathrm{j}}, v_{\mathrm{j}}\right)$ nach [8].

dreiecks $\triangle \mathrm{CDE}$ für jedes Gewinde eindeutig bestimmt. Ausgehend von der Lage des Ausgangsdreiecks $\triangle \mathrm{CDE}$ in der $\mathrm{x}$-z-Ebene des Werkstückkoordinatensystems $\mathrm{O}$ in Abb. 2 entsteht das Gewindeprofil im Axialschnitt A - A durch das Verschrauben des Ausgangsdreiecks. Das geometrisch-ideale Formelement in Abb. 1 setzt sich somit aus mehreren Regelschraubflächen (Abb. 3) zusammen. Jedes Hauptachsensystem der Regelschraubflächen besitzt ohne Zwangsbedingungen sechs Freiheitsgrade im Werkstückkoordinatensystem. Die insgesamt sechs Freiheitsgrade sind die Lageparameter $\left(t_{\mathrm{xj}}, t_{\mathrm{yj}}, t_{\mathrm{zj}}\right)$ für die Verschiebung und die Orientierungsparameter $\left(a_{\mathrm{xj}}, a_{\mathrm{yj}}, a_{\mathrm{zj}}\right)$ für die Drehungen um die Koordinatenachsen. Für die Eindeutigkeit des Modells müssen die sechs Freiheitsgrade anhand der Gewindegeometrie vollständig bestimmt sein. Die erste Bedingung ist, dass die $\mathrm{z}_{\mathrm{j}}$-Achsen fluchtend mit der $\mathrm{z}$-Achse sind. Mit dieser Einschränkung sind insgesamt vier Freiheitsgrade einer jeden Regelschraubfläche bestimmt. Hierbei handelt es sich um die translatorischen Freiheitsgrade $\left(t_{\mathrm{xj}}, t_{\mathrm{yj}}\right)$ und die rotatorischen Freiheitsgrade $\left(a_{\mathrm{xj}}, a_{\mathrm{yj}}\right)$. Die Rotationsfreiheitsgrade $a_{\mathrm{zj}}$ sind mit der Bedingung bestimmt, dass die $x_{\mathrm{j}}$ - $z_{\mathrm{j}}$-Ebenen koplanar mit derjenigen Ebene sind, in der das Ausgangsdreieck $\triangle \mathrm{CDE}$ definiert ist. Die letzten Translationsfreiheitsgrade $t_{\mathrm{zj}}$ ergeben sich aus der Bedingung, dass der Schnittpunkt $\mathrm{C}$ Teil beider Regelschraubflächen ist. An einem eingängigen Gewinde ergeben sich nach Abb. 1 folgende Bestimmungsgrößen:

Einzelflankensteigung $P_{\mathrm{Z} 1}, P_{\mathrm{Z} 2}$ 
- Kinematische Steigung $P_{\mathrm{ZT}}$

- Gewindeflankenwinkel $a_{1}, a_{2}$

- Gewindeprofilwinkel $a_{\mathrm{T}}$

- Kegelerzeugungswinkel $\varphi$

- Flankendurchmesser $d_{2}(z)$

Alle Gleichungen zur Berechnung der Bestimmungsgrößen aus den freien Parametern $\left(p_{\mathrm{j} 1}, p_{\mathrm{j} 2}, t_{\mathrm{zj}}\right)$ sind in [7] angegeben. auf einer Messspur beträgt $\Delta v_{j}=0,0166$ rad. Dadurch werden alle Messpunkte gleichmäßig auf der Gewindeoberfläche verteilt, so dass während der Auswertung eine einheitliche Gewichtung vorliegt. Die Robustheit der Messstrategie gegenüber variierenden Bedienern und KMG wird anhand der Streuung von vier Kalibrierungen untersucht, welche von drei Anwendern auf drei verschiedenen KMG durchgeführt wurden (Abschnitt 5).

\section{Auswertealgorithmus}

\section{Messstrategie}

Die neuartige Messstrategie ist weitestgehend automatisiert, um den potentiellen Einfluss durch variierende Bediener zu minimieren. Flächenhafte Messungen erfolgen auf Koordinatenmessgeräten (KMG) mit integriertem Drehtisch (RT) im Scanning-Modus. Die Messstrategie basiert auf den folgenden Schritten [8]:

1. Bestimmung des Werkstückkoordinatensystems (x,y,z) (Abb. 1) und Schnittpunkt C (Abb. 2) zur Definition der Ebene bei Beginn der Messung

2. Erstellung einer Soll-Punktewolke in einem externen Programm auf Basis des zuvor definierten Formelements in Parameterdarstellung (Gl. 1) unter Berücksichtigung der in 1. gemessenen Koordinaten und nominalen Bestimmungsgrößen (Abschnitt 2.2).

3. Import der Soll-Punktewolke in die KMGSteuersoftware

4. Ermittlung von Position und Orientierung der RTAchse

5. Abtastung der Gewindehelices in Einflankenanlage für die unabhängige flächenhafte Erfassung von Flanke 1 und Flanke 2 in einem gemeinsamen Bezugssystem

6. Messung auf Umschlag zur Reduzierung des RTEinflusses

7. Rückführung auf die SI-Einheit durch Substitutionsmessungen an Normalen

In den hier vorgestellten Beispielmessungen wird die Oberfläche der Flanken über die gesamte Länge der Gewindehelix erfasst. Bereiche, in denen sich Anschnitte befinden, werden jedoch ausgespart, so dass insgesamt neun volle Umdrehungen gemessen werden. Pro Kalibrierung werden insgesamt 81600 Punkte aufgenommen. Die Oberfläche der zwei Flanken wird mit jeweils sechs Spuren abgescannt. In radialer Richtung beträgt der Abstand zwischen den gemessenen Spuren $\Delta u_{j}=0,5 \mathrm{~mm}$. Das entsprechende Winkelinkrement zwischen zwei benachbarten Punkten
Der ganzheitliche Auswertealgorithmus liefert im ersten Schritt die Lösung des besteingepassten Formelements in die Ist-Punktewolke mit den Parametern $\left(p_{\mathrm{j} 1}, p_{\mathrm{j} 2}, t_{\mathrm{zj}}\right)$. Somit können die Abweichungen für die Bestimmungsgrößen am Gewinde und die Lage bezüglich der vordefinierten Nenngeometrie bestimmt werden. Des Weiteren liefern die finalen Residuen der Besteinpassung die Formabweichungen bezüglich der geometrisch-idealen Modellierung in Abschnitt 2. Der 3D-Bestfit-Algorithmus arbeitet nach den Ansätzen in [9]. Neue Betrachtungen zur Auswertung

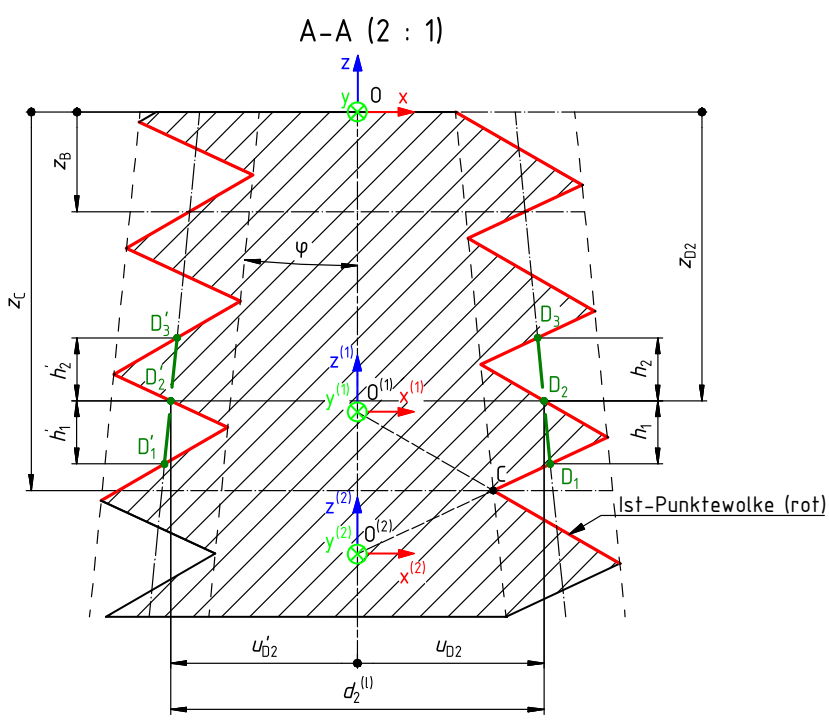

Abb. 4: Axialschnitt A-A des geometrisch-idealen Formelements mit der Betrachtung von lokalen Bestimmungsgrößen an der lstPunktewolke nach $[4,7]$.

von Bestimmungsgrößen am Gewinde liefert die Arbeit in [4]. Hier sind sogenannte lokale Bestimmungsgrößen definiert, welche zur Berechnung die Berührpunkte der IstPunktewolke als wirkliche Form eines Gewindes verwenden. Damit gelingt es, den Einfluss der Formabweichungen auf die Bestimmungsgrößen quantitativ auszudrücken. Die 
Ermittlung von lokalen Bestimmungsgrößen liefert einen wichtigen Beitrag zur Ermittlung des Paarungsflankendurchmessers eines Gewindes nach [4]. In dem vorliegenden Beitrag liegt der Fokus auf der Ermittlung der lokalen Flankendurchmesser $d_{2}^{(\mathrm{l})}(\mathrm{z})$. Die Berechnung der lokalen Flankendurchmesser baut auf das besteingepasste geometrisch-ideale Formelement in Abb. 4 auf. Für die lokalen Flankendurchmesser sind die sogenannten Flankendurchmesserlinien an der wirklichen Form des Gewindes gesucht. Jeder lokale Flankendurchmesser berechnet sich aus den Radialkoordinaten $\left(u_{\mathrm{D} 2}, u_{\mathrm{D} 2}^{\prime}\right)$ der Flankendurchmesserlinien nach

$$
d_{2}^{(1)}(z)=u_{\mathrm{D} 2}(z)+u_{\mathrm{D} 2}^{\prime}(z) .
$$

Gl. 2 lässt sich anhand Abb. 4 ableiten. In Abb. 4 sind zwei gegenüberliegende Flankendurchmesserlinien, die Strecken $\overline{\mathrm{D}_{1} \mathrm{D}_{3}}$ und $\overline{\mathrm{D}_{1}^{\prime} \mathrm{D}_{3}^{\prime}}$, eingezeichnet, wobei die Punkte $\mathrm{D}_{\mathrm{i}}$ Berührpunkte darstellen und Teil der Ist-Punktewolke sind. Für die einzelnen Flankendurchmesserlinien gilt, dass die Abschnitte bestehend aus Gewindezahn (z. B. Strecke $\overline{\mathrm{D}_{1} \mathrm{D}_{2}}$ ) und Gewindelücke (z. B. Strecke $\overline{\mathrm{D}_{2} \mathrm{D}_{3}}$ ) gleich lang sind. Somit ergeben sich die Bedingungen nach Gl. 3 für die jeweils paarweisen Flankendurchmesserlinien

$$
h_{1} \stackrel{!}{=} h_{2} \quad h_{1}^{\prime} \stackrel{!}{=} h_{2}^{\prime} .
$$

Aus den genannten Bedingungen folgt eine eindeutige radiale Lage $\left(u_{\mathrm{D} 2}, u_{\mathrm{D} 2}^{\prime}\right)$ der Flankendurchmesserlinien. Mit dieser Methode sind nach Gl. 2 die lokalen Flankendurchmesser in Richtung der z-Achse des Werkstückkoordinatensystems zu bestimmen. Die zugehörige $\mathrm{z}_{\mathrm{D} 2}$-Koordinate ist als Schwerpunktkoordinate der sechs z-Koordinaten von den Berührpunkten $\mathrm{D}_{\mathrm{i}}$ und $\mathrm{D}_{\mathrm{i}}^{\prime}$ mit $i=1,2,3$ zu berechnen.

\section{Messergebnisse}

Es werden Ergebnisse aus Kalibrierungen eines Gewindelehrdorns M64x6 vorgestellt, die sich auf die lokalen Flankendurchmesser $d_{2}^{(\mathrm{l})}(\mathrm{z})$ (Gl. 2) und die entsprechende Lösung nach der Methode der kleinsten Quadrate $d_{2}(\mathrm{z})$ beschränken. Darüber hinaus wird für die Abschätzung des Bedienereinflusses die Streuung des lokalen Flankendurchmesser untersucht. Anschließend folgt ein quantitativer Vergleich mit simulierten Unsicherheiten. Eine ausführliche Darstellung aller ermittelten Gewindeparameter ist in [6], [7] und [8] gegeben.

Abb. 5 zeigt Auswerteergebnisse für den Flankendurchmesser nach der Methode der kleinsten Quadrate (Geraden) und den lokalen Flankendurchmesser (Punkte) aus vier Kalibrierungen (M1 - M4), die auf drei verschiedenen KMG durch drei Bediener durchgeführt wurden, aufgetragen über die Werkstückkoordinate z in Axialrichtung des Gewindes. Es wird darauf hingewiesen, dass es sich bei sämtlichen dargestellten Daten (Geraden und Punkte) um Einpassungen handelt. Der Nominalwert 60, 103 mm [5] wird von allen ermittelten Ergebnissen zwischen minimal $13 \mu \mathrm{m}$ und maximal $16 \mu \mathrm{m}$ überschritten. Die bestimmten Flankendurchmesser werden ohne Ausnahme mit zunehmender z-Koordinate über den gesamten Messbereich um ca. $3 \mu \mathrm{m}$ kleiner. Daher liegt am untersuchten Gewinde eine leichte Kegeligkeit vor.

Die Resultate aller Messungen weisen zwischen $d_{2}^{(\mathrm{l})}(\mathrm{z})$ und $d_{2}(\mathrm{z})$ einen Offset auf, der gemittelt über die z-Koordinate $-4,5 \mu \mathrm{m}$ beträgt. Diese Differenz lässt sich anhand konkav geformter Flanken im Bereich des Flankendurchmessers erklären, welche durch weitere Untersuchungen nachgewiesen wurden [8]. Erst die gemeinsame Analyse von $d_{2}(\mathrm{z})$ und $d_{2}^{(\mathrm{l})}(\mathrm{z})$ ermöglicht den Rückschluss auf vorliegende Formabweichungen.

Die Standardabweichung $\sigma$ von $d_{2}^{(1)}(\mathrm{z})$ beträgt gemittelt über die z-Koordinate $0,1 \mu \mathrm{m}$. In [8] erfolgt eine Abschätzung der Scanning-Unsicherheit $U_{S} \approx 0,2 \mu \mathrm{m}$ und der Unsicherheit aus dem Messprozess $U_{P} \approx 1 \mu \mathrm{m}$ mit Hilfe von Monte-Carlo Simulationen. Die experimentell ermittelte Standardabweichung ist damit halb so groß wie $U_{S}$ und signifikant kleiner als $U_{P}$. Durch die weitestgehend automatisierte Messstrategie ist der Bedienereinfluss in Relation zu anderen Unsicherheitsbeiträgen als gering einzuordnen.

\section{Zusammenfassung und Ausblick}

Es wurde ein 3D-Kalibrierverfahren zur Ermittlung von Bestimmungsgrößen am Gewinde unter der Verwendung von fortschrittlichsten Technologien in der Koordinatenmesstechnik entwickelt. Alle Aspekte der neuartigen Kalibriermethode sind darauf ausgerichtet, die komplexe Gewindegeometrie erstmals vollständig zu erfassen, so dass eine funktionsgerechte Prüfung erfolgen kann.

Das präsentierte 3D-Modell der Gewindegeometrie wird in Form von Soll-Antastpunkten in die Steuerungssoftware integriert und bildet damit sowohl die Basis für die Messstrategie als auch für den Auswertealgorithmus.

Die Betrachtung der lokalen Gewindeparameter ermöglicht erstmals die Ermittlung von Formabweichungen, die durch konventionelle 2D-Kalibrierungen nicht abgebildet werden.

Insbesondere führt der hohe Automatisierungsgrad dazu, 


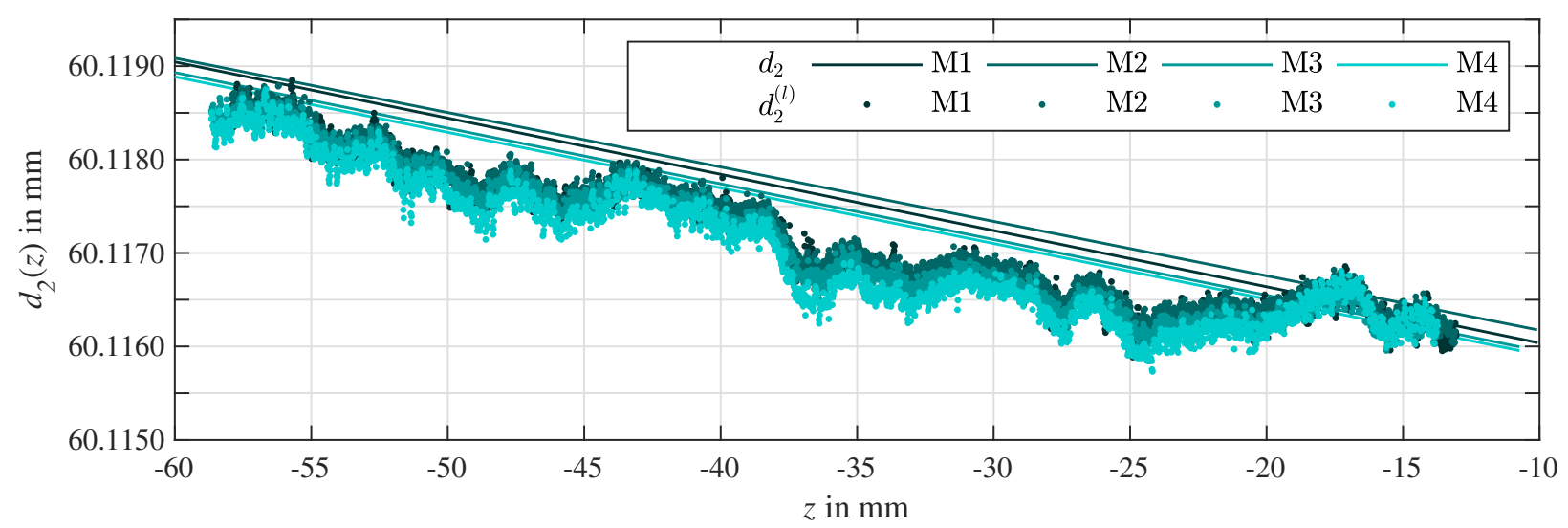

Abb. 5: Auswerteergebnisse für vier verschiedene Kalibrierungen (M1-M4) eines Gewindelehrdorns M64×6. Dargestellt sind ermittelte Flankendurchmesser nach der Methode der kleinsten Quadrate $d_{2}(\mathrm{z})$ (Geraden) und lokale Flankendurchmesser $d_{2}^{(1)}(\mathrm{z})(\mathrm{Punkte})$, aufgetragen über der Gewindeachse z. Es wird darauf hingewiesen, dass $d_{2}(\mathrm{z})$ nicht die Einpassung von $d_{2}^{(1)}(\mathrm{z})$ ist.

dass des Bedienereinfluss weitestgehend reduziert wird. Anhand der vorgestellten Ergebnisse wurde gezeigt, dass die Standardabweichung von Ergebnissen, die durch unterschiedliche Bediener hervorgerufen werden, signifikant kleiner sind als die ermittelte Unsicherheit aus dem Prozess.

Eine umfangreiche Verifikation des 3D-Kalibrierverfahrens wird in Kürze in [6] veröffentlicht.

Darüber hinaus erfolgt momentan die Erweiterung der Kalibriermethode zur Integration weiterer Flankenformen, so dass in Zukunft die funktionsgerechte 3D-Kalibrierung von wendelförmigen Maschinenelementen wie Schnecken und Verzahnungen auf Grundlage der vorgestellten Entwicklungen realisiert wird.

Danksagung: Die vorgestellten Arbeiten wurden vom Bundesministerium für Wirtschaft und Energie (BMWi) der Bundesrepublik Deutschland im Rahmen des Förderprogramms MNPQ-Transfer finanziert.

\section{Literatur}

[1] EURAMET cg-10. Determination of pitch diameter of parallel thread gauges by mechanical probing, 2012.

[2] M. Kochsiek und J. Lerch. Zur Ermittlung von Bestimmungsgrössen an Gewinden, Band PTB-Me-4 of PTB-Bericht : Abt. Mechanik. Physikalisch-Technische Bundesanstalt, Braunschweig, 1974.

[3] H. Kunzmann, J. Lerch und F. Wäldele. Messen von Gewinden auf rechnergesteuerten Koordinatenmeßgeräten. PTBMitteilungen, 90(6):434-441, 1980.

[4] T. Meß. Der Paarungsflankendurchmesser - Untersuchung des begrifflichen Umfeldes, der Einflussgrößen und der Bedeutung für die Funktion, Spezifikation, Fertigung und Prüfung von Gewinden. Dissertation, Technische Universität IImenau, Ilmenau, 2017.

[5] Norm DIN 13-1 1999-11. Metrisches ISO-Gewinde allgemeiner Anwendung - Teil 1: Nennmaße für Regelgewinde; GewindeNenndurchmesser von $1 \mathrm{~mm}$ bis $68 \mathrm{~mm}$. Beuth Verlag $\mathrm{GmbH}$, Berlin, 1999.

[6] A. Przyklenk, S. Schädel, A. Wedmann und M. Stein. Comprehensive verification of a novel $3 d$ calibration method with respect to a standardized $2 \mathrm{~d}$ thread pitch diameter procedure. 2020. In Vorbereitung zur Einreichung bei Measurement Science and Technology.

[7] S. Schädel. Neuartiges Messverfahren zur 3DGewindekalibrierung unter Verwendung einer flächenhaften Messstrategie und eines ganzheitlichen Auswertealgorithmus. Dissertation, Technische Universität Ilmenau, IImenau, 2020. Im Veröffentlichungsprozess.

[8] S. Schädel, A. Wedmann und M. Stein. Advanced screw thread metrology using an areal measuring strategy and a holistic evaluation method. Measurement Science and Technology, 2019. ISSN 0957-0233. 10.1088/1361-6501/ab1501.

[9] D. Sourlier und A. Bucher. Normgerechter Bestfit-Algorithmus für Freiformflächen oder andere nicht-reguläre Ausgleichsflächen in Parameterform / Exact bestfit algorithm applicable to sculptured surfaces or to any non-regular surfaces in parametric form. tm - Technisches Messen, 59(7-8), 1992. ISSN 0171-8096. 10.1524/teme.1992.59.78.293. 